\title{
Adaptive Compensation Method Based on STCKF for AHRS
}

\author{
Shengfeng $\mathrm{Gao}^{\mathrm{a}}$, Hai Zhu ${ }^{\mathrm{b}}$, Zhengdong $\mathrm{Guo}^{\mathrm{c}}$ \\ Navy Submarine Academy, Qingdao 266199, China \\ agaoshengfeng205@126.com, bzhuhai123@126.com, cg_zh_d@aliyun.com
}

Keywords: AHRS, electronic compass, magnetic deviation, STCKF, adaptive compensation.

\begin{abstract}
Aimed at the problem that the compensation method for attitude and heading reference system (AHRS) cannot adapt the changing magnetic field environment, an adaptive compensation method based on strong tracking cubature Kalman filter (STCKF) is putted forward. The system equation of magnetic deviation model is gave out by Poisson equation.With reference to the strong tracking filter (STF), a strong tracking cubature Kalman filter (STCKF) is proposed by introducing a time-varying fading factor to estimate the model parameter in real time. Then adaptive compensation is realized. The results show that adaptive compensation method based on STCKF can adapt the changing magnetic field environment very well and have the higher precision than the compensation method using Kalman filter (KF).
\end{abstract}

\section{Introduction}

Along with the development of ocean industry, UUV (Unmanned Underwater Vehicle) with the small volume and invisibility, as the effective tool to explore and develop the ocean, gets the extensive use. In order to ensure the effectiveness of the data it collected, the navigation information such as the position and heading etc of UUV must be known accurately. Thus the ability of accurate positioning is the basic essential factor for UUV to carry out the assignment under water successfully.

Thanks to the development of MEMS (micro electronic mechanical system), the AHRS (attitude and heading reference system), which is consisted of the micro gyro, accelerometer and electronic compass has became one of the most important navigation means of the UUV. The electronic compass is critical factor that influences the precision of the system heading. However, the magnetometer has manufacturing properties error and the assembing error in the production course, as well as the work environment of electronic compass is fairly odious because of the influence that power supply noise, outer magnetic field interference and the surrounding ferromagnetic material and so on. It makes the electronic compass has error unavoidablly, which is called the magnetic deviation[1].

At present there are two kinds of compensation method of magnetic deviation. The first one is using the external information,such as GPS, rotary tables etc to compensate the magnetic deviation[2-4]. This method has the higher precision,but the lower suitability. The second one is attitude-independent magnetometer calibration. Gambhir applies the centered algorithm to determine magnetometer biases to yield a quadratic loss function that can be minimized using simple linear least aquares[5].Alonso and Shuster develop the TWOSTEP algorithm to determine magnetometer biases without knowledge of the attitude[6]. And lots of algorithms have been propsed based on ellipsoid surface fitting for estimating the magnetic deviation[7-9].This method doesn't need the external information of the attitude,but the quality of sampling point must be superior. However, both of the methods are only suit for the inital compensation of the electronic compass. The magnetic field environment around the electronic compass is always changing, just like the magnetic field of the electronic equipment carried on the UUV, the eddy current magnetic field of the UUV etc. All of those would influence the precision of the electronic compass. Thus the electronic compass should be compensated in real-time. Feng Yi-bo apply the real-time compensation method for heading error of electronic compass using Kalman filter (KF)[10]. But the calibration precision is much lower, so that an higher precision adaptive compensation method is needed. This paper develop magnetic deviation model of Poisson equation, and a strong tracking cubature Kalman filter (STCKF) is proposed by 
introducing a time-varying fading factor to filtering process with reference to the strong tracking filter (STF).

\section{The Measurement Model}

The electronic compass of attitude and heading reference system (AHRS) calculates the heading of the UUV by measuring the geomagnetic field. Because of the manufacturing properties error and the assembing error of magnetometer in the production course, it makes the electronic compass has measurement error unavoidablly. In this section the three- axis magnetometers (TAM) measurement model is summarized by Poisson equation[11].

$$
B=K A^{-1} H_{g}+H_{I}
$$

Where $B=\left[\begin{array}{lll}B_{x} & B_{y} & B_{z}\end{array}\right]^{T}$ is the measurement of the magnetic field by the magnetometer, $H_{g}=\left[\begin{array}{lll}H_{g x} & H_{g y} & H_{g z}\end{array}\right]^{T}$ is the corresponding value of the geomagnetic field with respect to an Earth-fixed coordinate system, $H_{I}=\left[\begin{array}{lll}H_{I x} & H_{I y} & H_{I z}\end{array}\right]^{T}$ is the bias vector, $K=\left[\begin{array}{ccc}1+a & b & c \\ d & 1+e & f \\ g & h & 1+k\end{array}\right]$ is the scale factors, and $A^{-1}=\left[\begin{array}{ccc}\cos \theta & \sin \gamma \sin \theta & \cos \gamma \sin \theta \\ 0 & \cos \gamma & -\sin \gamma \\ -\sin \theta & \sin \gamma \cos \theta & \cos \gamma \cos \theta\end{array}\right]$ is the attitude matrix of the magnetometer with respect to the Earth-fixed coordinate.

The AHRS cannot provide the accurate heading, when it is in deviation calibrating stage. So the true attitude matrix of the magnetometer with respect to the Earth-fixed coordinate $A_{\text {true }}^{-1}$ is as follow

$$
A_{\text {true }}^{-1}=\left[\begin{array}{ccc}
\cos \psi_{e} & -\sin \psi_{e} & 0 \\
\sin \psi_{e} & \cos \psi_{e} & 0 \\
0 & 0 & 1
\end{array}\right] \cdot A^{-1}
$$

Where $\psi_{e}$ is the heading misalignment angle.

Then select the model parameter and the heading misalignment angle as the state variable of the filter, as follow

$$
X=\left[\begin{array}{lllllllllllll}
a+1 & b & c & d & e+1 & f & g & h & k+1 & H_{I x} & H_{I y} & H_{I z} & \psi_{e}
\end{array}\right]
$$

Because the state variable are all constant, so that the state model is given by

$$
X(k+1)=X(k)+W(k)
$$

Where $W(k)$ is assumed to be a zero-mean Gaussian process with covariance $Q_{W}(k)$.

According to Eq. (1), select the measurement of the magnetic field by the magnetometer as the measurement variable of the filter. And the measurement model is given by

$$
Z(k)=h(X(k))+V(k)
$$

Where $V(k)$ is assumed to be a zero-mean Gaussian process with covariance $R_{V}(k)$.

According to Eq. (1) and Eq. (2), we can get $h(X(k))$

$$
\begin{aligned}
h_{1}(X)= & (a+1)\left[\left(A_{11}^{-1} \cos \psi_{e}-A_{12}^{-1} \sin \psi_{e}\right) H_{g x}+\left(A_{11}^{-1} \sin \psi_{e}+A_{12}^{-1} \cos \psi_{e}\right) H_{g y}+A_{13}^{-1} H_{g z}\right]+ \\
b & {\left[\left(A_{21}^{-1} \cos \psi_{e}-A_{22}^{-1} \sin \psi_{e}\right) H_{g x}+\left(A_{21}^{-1} \sin \psi_{e}+A_{22}^{-1} \cos \psi_{e}\right) H_{g y}+A_{23}^{-1} H_{g z}\right]+} \\
c & {\left[\left(A_{31}^{-1} \cos \psi_{e}-A_{32}^{-1} \sin \psi_{e}\right) H_{g x}+\left(A_{31}^{-1} \sin \psi_{e}+A_{32}^{-1} \cos \psi_{e}\right) H_{g y}+A_{33}^{-1} H_{g z}\right]+H_{I x} }
\end{aligned}
$$




$$
\begin{aligned}
& h_{2}(X)= d\left[\left(A_{11}^{-1} \cos \psi_{e}-A_{12}^{-1} \sin \psi_{e}\right) H_{g x}+\left(A_{11}^{-1} \sin \psi_{e}+A_{12}^{-1} \cos \psi_{e}\right) H_{g y}+A_{13}^{-1} H_{g z}\right]+ \\
&(e+1)\left[\left(A_{21}^{-1} \cos \psi_{e}-A_{22}^{-1} \sin \psi_{e}\right) H_{g x}+\left(A_{21}^{-1} \sin \psi_{e}+A_{22}^{-1} \cos \psi_{e}\right) H_{g y}+A_{23}^{-1} H_{g z}\right]+ \\
& f\left[\left(A_{31}^{-1} \cos \psi_{e}-A_{32}^{-1} \sin \psi_{e}\right) H_{g x}+\left(A_{31}^{-1} \sin \psi_{e}+A_{32}^{-1} \cos \psi_{e}\right) H_{g y}+A_{33}^{-1} H_{g z}\right]+H_{I y} \\
& h_{3}(X)=g\left[\left(A_{11}^{-1} \cos \psi_{e}-A_{12}^{-1} \sin \psi_{e}\right) H_{g x}+\left(A_{11}^{-1} \sin \psi_{e}+A_{12}^{-1} \cos \psi_{e}\right) H_{g y}+A_{13}^{-1} H_{g z}\right]+ \\
& h\left[\left(A_{21}^{-1} \cos \psi_{e}-A_{22}^{-1} \sin \psi_{e}\right) H_{g x}+\left(A_{21}^{-1} \sin \psi_{e}+A_{22}^{-1} \cos \psi_{e}\right) H_{g y}+A_{23}^{-1} H_{g z}\right]+ \\
&(k+1)\left[\left(A_{31}^{-1} \cos \psi_{e}-A_{32}^{-1} \sin \psi_{e}\right) H_{g x}+\left(A_{31}^{-1} \sin \psi_{e}+A_{32}^{-1} \cos \psi_{e}\right) H_{g y}+A_{33}^{-1} H_{g z}\right]+H_{I z}
\end{aligned}
$$

The measurement model is clearly nonlinear in the state variable $X$. Therefore, linear least squares cannot be applied directly. An adaptive compensation method based on strong tracking cubature Kalman filter (STCKF) is putted forward in this paper.

\section{Strong Tracking Cubature Kalman Filter(STCKF)}

\subsection{Cubature Kalman Filter.}

The basic thought of Cubature Kalman Filter (CKF) is to transform the nonlinear filter to the integration of nonlinear function and Gaussian probability-density [12]. The nonlinear system is given by

$$
\begin{aligned}
& x_{k}=f\left(x_{k-1}\right)+w_{k-1} \\
& z_{k}=h\left(x_{k}\right)+v_{k}
\end{aligned}
$$

Where $x_{k} \in R^{n}$ is the state variable at time $k, z_{k} \in R^{m}$ is the measurement, $w_{k-1}$ is the state noise vector that is assumed to be a zero-mean Gaussian process with covariance $Q_{k-1}$, and $v_{k}$ is the state noise vector that is assumed to be a zero-mean Gaussian process with covariance $R_{k}$.

First, CKF calculate the cubature point and its weight

$$
\xi_{j}=\sqrt{\frac{m}{2}}[1]_{j}, \omega_{j}=\frac{1}{m}
$$

Where $m$ is the totality of the cubature points, and $m=2 n$ in Spherical-Radial Cubature law ( $n$ is the dimension of the system state). [1] $=[$ eye $(n),-$ eye $(n)]$ is an $n \times 2 n$ matrix. If the posterior probability, $p\left(x_{k} \mid z_{k}\right) \sim N\left(x_{k} ; \hat{x}_{k}, P_{k}\right)$ follow the Gauss distribution at time $k$, and $S_{k}$ is the Cholesky factor of $P_{k}$. Then Cubature Kalman Filter (CKF) is just as follow

(1) State estimate extrapolation:

The cubature points are calculated by

$$
X_{j, k-1}=S_{k-1} \xi_{j}+\hat{x}_{k-1}
$$

The transformed set of cubature points are evaluated for each of the points by

$$
X_{j, k}^{*}=f\left(X_{j, k-1}\right)
$$

The predicted state estimate and covariance are given by

$$
\begin{aligned}
& \bar{X}_{k}=\sum_{j=1}^{m} \omega_{j} X_{j, k}^{*} \\
& \bar{P}_{k}=\sum_{j=1}^{m} \omega_{j} X_{j, k}^{*} X_{j, k}^{* T}-\bar{x}_{k} \bar{x}_{k}^{T}+Q_{k-1}
\end{aligned}
$$

(2) Observation update:

The Cholesky factor of $\bar{P}_{k}$ is given by

$\bar{P}_{k}=\bar{S}_{k} \bar{S}_{k}^{T}$

The cubature points are calculated by

$$
X_{j, k}=\bar{S}_{k} \xi_{j}+\bar{x}_{k}
$$


The transformed set of cubature points are evaluated for each of the points by

$Z_{j, k}=h\left(X_{j, k}\right)$

The predicted observation estimate, output covariance and cross correlation matrix are given by

$$
\begin{aligned}
& \bar{Z}_{k}=\sum_{j=1}^{m} \omega_{j} Z_{j, k} \\
& P_{z z, k}=\sum_{j=1}^{m} \omega_{j} Z_{j, k} Z_{j, k}^{T}-\bar{Z}_{k} \bar{Z}_{k}^{T}+R_{k} \\
& P_{x z, k}=\sum_{j=1}^{m} \omega_{j} X_{j, k} Z_{j, k}^{T}-\bar{x}_{k} \bar{Z}_{k}^{T}
\end{aligned}
$$

The estimate of gain matrix, state and error covariance are given by

$$
\begin{aligned}
& W_{k}=P_{x z, k} P_{z z, k}^{-1} \\
& \hat{x}_{k}=\bar{x}_{k}+W_{k}\left(z_{k}-\bar{z}_{k}\right) \\
& P_{k}=\bar{P}_{k}-W_{k} P_{z z, k} W_{k}^{T}
\end{aligned}
$$

Compared with other nonlinear filter, CKF use definite sample points to calculate the probability density function instead of calculating the Jacobin matrix. So it can be realized more easily and has higher filtering precision.

\subsection{Strong Tracking Cubature Kalman Filter.}

Although CKF realize more easily and have higher filtering precision, but its tracking ability to the mutational status is much worse. Then Strong Tracking Filter (STF)[13] is leaded in CKF to improve the adaptation of filter for the mutational status.

STF bring a time-varying fading factor in the filter. The factor can adjust the system gain matrix on line to compel the output errors mutually orthogonal, so that the filter can track the mutational status adaptively.

For the state estimate of the system

$$
\hat{X}_{k+1}=\hat{X}_{k+1 \mid k}+K_{k+1}\left(Z_{k+1}-\hat{Z}_{k+1 \mid k}\right)
$$

Orthogonality principle is the sufficient condition of the filter to be STF. Also select a time-varying gain matrix $K_{k+1}$ to make

$$
\begin{aligned}
& E\left[X_{k+1}-\hat{X}_{k+1}\right]\left[X_{k+1}-\hat{X}_{k+1}\right]^{T}=\min \\
& E\left[v_{k+1+j} v_{k+1}^{T}\right]=0, \quad k=0,1,2, \cdots, j=1,2, \cdots
\end{aligned}
$$

Eq. (21) request the output errors to be orthogonal at different time.

In the real filtering process, when the system occur much larger uncertainty, the output errors are not mutually orthogonal. In order to keep the specialty, a time-varying fading factor should be brought in the predicted covariance to reduce the influence of the past data.

One of the suboptimum fading factors is given by [13]

$$
\lambda_{k+1}=\left\{\begin{array}{cc}
\eta_{k+1} & \eta_{k+1}>1 \\
1 & \eta_{k+1} \leq 1
\end{array}\right.
$$

Where $\eta_{k+1}=\frac{\operatorname{tr}\left[N_{k+1}\right]}{\operatorname{tr}\left[M_{k+1}\right]}$, and $N_{k+1}, M_{k+1}$ are given by

$$
\begin{aligned}
& N_{k+1}=V_{0, k+1}-\beta R_{k+1}-H_{k+1} Q_{k+1} H_{k+1}^{T} \\
& M_{k+1}=H_{k+1} \Phi P_{k+1} \Phi^{T} H_{k+1}^{T}
\end{aligned}
$$

Where $\beta$ is the priori coefficient, and $\beta \geq 1$. $V_{0, k+1}$ is the error covariance matrix and is given by 


$$
\mathrm{V}_{0, \mathrm{k}+1}=E\left[v_{k+1} v_{k+1}^{T}\right]=\left\{\begin{array}{cc}
v_{1} v_{1}^{T} & k=0 \\
\frac{\rho V_{0, k}+v_{k+1} v_{k+1}^{T}}{1+\rho} & k \geq 1
\end{array}\right.
$$

Where $\rho$ is the forgetting factor, and $0<\rho \leq 1$, generally $\rho=0.95$.

According to above process, STF using the current measurement of the model information to optimize the gain matrix $K_{k+1}$, so that the innovation can be mutually orthogonal. The adaptation of STF mainly reflects on the predicted covariance of the model. Therefore a time-varying fading factor is brought in CKF to adjust the system predicted covariance on line, so that the filter gain matrix can modulate adaptively.

Then the predicted covariance from Eq. (13) become to

$$
\bar{P}_{k}=\lambda_{k}\left(\sum_{j=1}^{m} \omega_{j} X_{j, k}^{*} X_{j, k}^{* T}-\bar{x}_{k} \bar{X}_{k}^{T}\right)+Q_{k-1}
$$

However, CKF is based on the Spherical-Radial Cubature law. It does not need to calculate the Jacobin matrix. Then we can get the suboptimum fading factor $\lambda_{k}$ as follow

$$
\begin{aligned}
& \mathrm{V}_{0, \mathrm{k}}=E\left[v_{k} v_{k}^{T}\right]=\left\{\begin{array}{cc}
v_{1} v_{1}^{T} & k=1 \\
\frac{\rho V_{0, k-1}+v_{k} v_{k}^{T}}{1+\rho} & k \geq 2
\end{array}\right. \\
& N_{k}=V_{0, k}-R_{k}-Q_{k} \\
& M_{k}=P_{x x, k}=\sum_{j=1}^{m} \omega_{j} h\left(X_{j, k}^{*}\right) h^{T}\left(X_{j, k}^{*}\right)-\bar{x}_{k} \bar{x}_{k}^{T} \\
& \eta_{k}=\frac{\operatorname{tr}\left[N_{k}\right]}{\operatorname{tr}\left[M_{k}\right]} \\
& \lambda_{k}=\left\{\begin{array}{cc}
\eta_{k} & \eta_{k}>1 \\
1 & \eta_{k} \leq 1
\end{array}\right.
\end{aligned}
$$

Just like the CKF, if we instead the Eq. (13) to Eq. (26), we can get Strong Tracking Cubature Kalman Filter (STCKF).

\section{Experimental Results}

In this section results of the Fixed Parameter [9], KF [10] and STCKF formulations are shown using experimental data. The AHRS (MTI-G-710) is fixed on the nonmagnetic platform coaxially. The experimental data are compared with the data from GPS. This paper is using root-mean-square error (RMSE) as the evaluation indicator of the compensation effect, which is given by

$$
\sigma=\sqrt{\frac{1}{n} \sum_{i=1}^{n}\left(x_{i}-x_{\text {true }}\right)^{2}}
$$

Where $x_{i}$ is the measurement, $x_{\text {true }}$ is the reference value and $n$ is the total number of measurements.

\subsection{Experiment System.}

The experiment system is constituted by nonmagnetic platform, AHRS (MTI-G-710), GPS, laptop and proton magnetometer. The system is shown in Fig. 1 . The briquette is fixed on the both side of the platform in order to apply ferromagnetic effect on AHRS. Data collection is using the soft MT Manager and the sample frequency is $400 \mathrm{~Hz}$. The geomagnetic field scalar quantity is measured by proton magnetometer. 


\subsection{Circumference Experiment.}

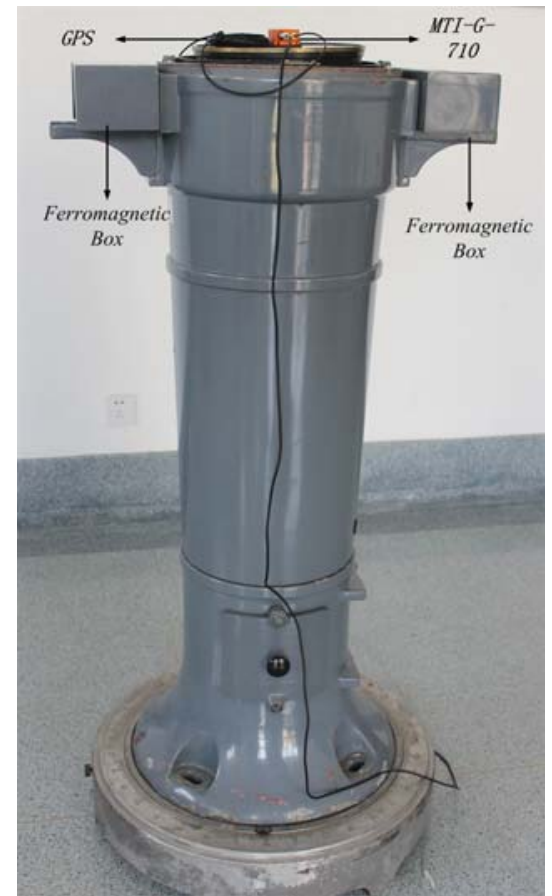

Fig. 1 Experiment system

Fix the AHRS on the nonmagnetic platform with the $Z$ axis upward. Then revolve the platform. The two dimensional curve of the $X$ axis and $Y$ axis measurement of the magnetometer should be a ellipse and the curve after compensation should be a circle in ideal condition. The experimental data are treated with the Fixed Parameter, KF and STCKF, which are shown in Fig. 2. The results show that all of those compensation methods have good effect without changing magnetic disturbance.
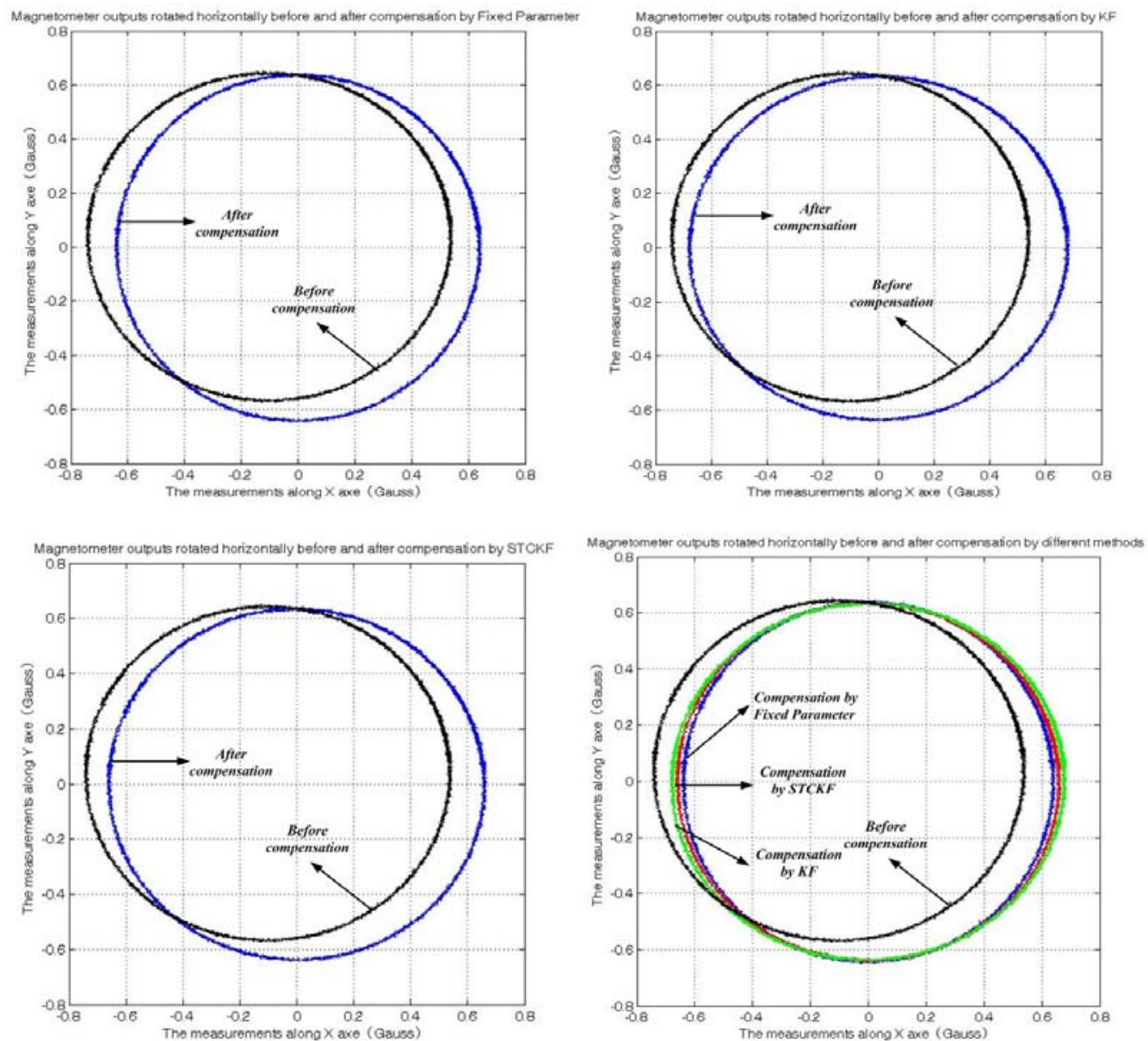

Fig. 2 Magnetic sensor outputs rotated horizontally in the earth's field before and after compensation 
Tab.1 The results of different compensation methods

\begin{tabular}{ccccccc}
\hline \multirow{2}{*}{$\left(^{\circ}\right)$} & \multicolumn{2}{c}{ Fixed Parameter } & \multicolumn{2}{c}{$\mathrm{KF}$} & \multicolumn{2}{c}{ STCKF } \\
\cline { 2 - 7 } & $\psi_{1}\left({ }^{\circ}\right)$ & $\Delta \psi_{1}\left(^{\circ}\right)$ & $\psi_{2}\left(^{\circ}\right)$ & $\Delta \psi_{2}\left(^{\circ}\right)$ & $\psi_{3}\left(^{\circ}\right)$ & $\Delta \psi_{3}\left(^{\circ}\right)$ \\
\hline 20 & 19.9276 & 0.0724 & 18.7789 & 1.2211 & 19.7999 & 0.2001 \\
40 & 40.1577 & -0.1577 & 38.2413 & 1.7587 & 39.7437 & 0.2563 \\
60 & 60.2649 & -0.2649 & 58.4224 & 1.5776 & 59.7327 & 0.2673 \\
80 & 80.0403 & -0.0403 & 79.1010 & 0.8990 & 80.0273 & -0.0273 \\
100 & 100.3968 & -0.3968 & 101.8014 & -1.8014 & 100.2015 & -0.2015 \\
120 & 119.1637 & 0.8363 & 121.5666 & -1.5666 & 120.3968 & -0.3968 \\
140 & 139.6999 & 0.3001 & 141.4107 & -1.4107 & 140.6103 & -0.6103 \\
160 & 159.3953 & 0.6047 & 161.5325 & -1.5325 & 160.1129 & -0.1129 \\
180 & 179.7082 & 0.2918 & 178.6675 & 1.3325 & 179.6905 & 0.3095 \\
200 & 199.9479 & 0.0521 & 198.0551 & 1.9449 & 199.5685 & 0.4315 \\
220 & 220.5613 & -0.5613 & 218.4949 & 1.5051 & 219.5991 & 0.4009 \\
240 & 240.3180 & -0.3180 & 238.3499 & 1.6501 & 239.5865 & 0.4135 \\
260 & 259.7223 & 0.2777 & 258.6702 & 1.3298 & 259.6291 & 0.3709 \\
280 & 280.6255 & -0.6255 & 281.0026 & -1.0026 & 280.3273 & -0.3273 \\
300 & 299.8283 & 0.1717 & 301.2684 & -1.2684 & 300.8207 & -0.8207 \\
320 & 320.0897 & -0.0897 & 321.8601 & -1.8601 & 320.7154 & -0.7154 \\
340 & 340.0495 & -0.0495 & 341.2587 & -1.2587 & 340.808 & -0.808 \\
360 & 359.4075 & 0.5925 & 358.5123 & 1.4877 & 359.4744 & 0.5256 \\
\hline
\end{tabular}

\begin{tabular}{ccccc}
\hline \multicolumn{5}{c}{ Tab.2 The RMSE of different compensation methods } \\
\hline & Uncompensated & Fixed Parameter & KF & STCKF \\
\hline RMSE $\left(^{\circ}\right)$ & 80.8247 & 0.3928 & 1.4927 & 0.4555
\end{tabular}

Tab. 1 and Tab. 2 shows that all of those compensation methods have good effect without changing magnetic disturbance. However, STCKF and Fixed Parameter have the nearly compensation effect, and much better than KF by comparing the RMSE of the three methods.

\subsection{Disturbance Experiment.}

Fix the AHRS on the nonmagnetic platform with the $Z$ axis upward and keep the $X$ axis to north, which means that the heading of AHRS is zero. Then change the magnetic field around AHRS. The measurements along the three axes of magnetometer are shown in Fig. 3. The results show that measurements along the three axes of magnetometer have a great fluctuation when the magnetic field environment changes. So the compensation model parameters should be adjusted along with the changing magnetic field environment in order to improve the compensation precision.

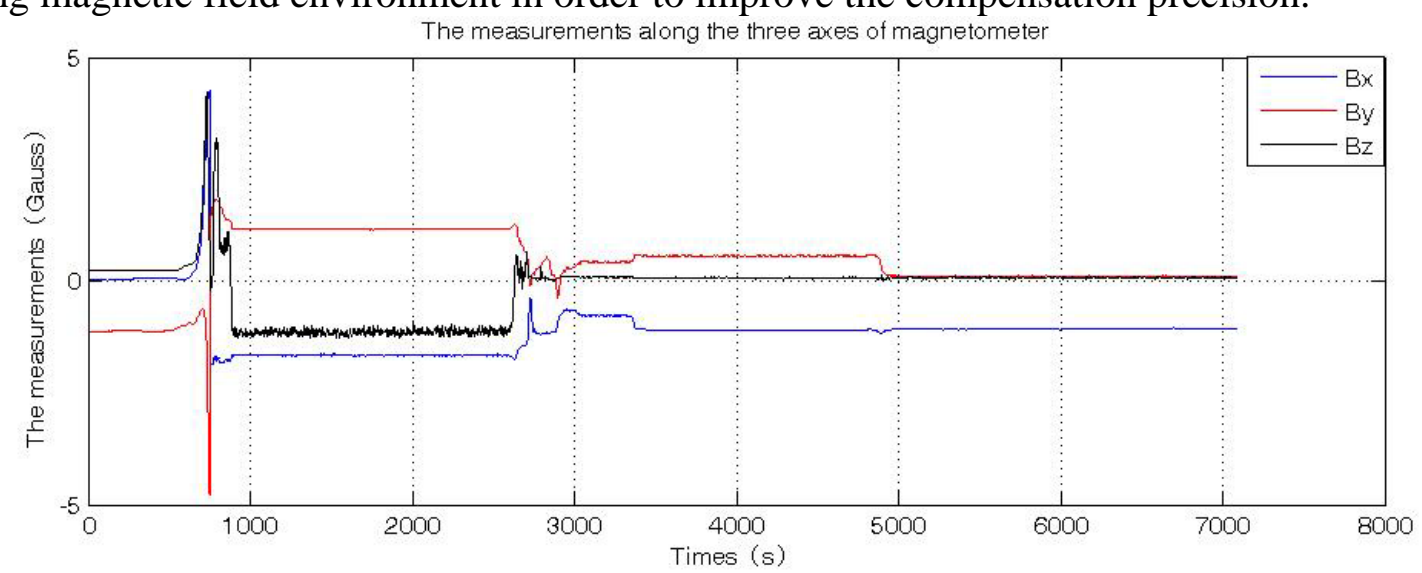

Fig.3 The axial intensity of magnetic field along the three axes of magnetometer 


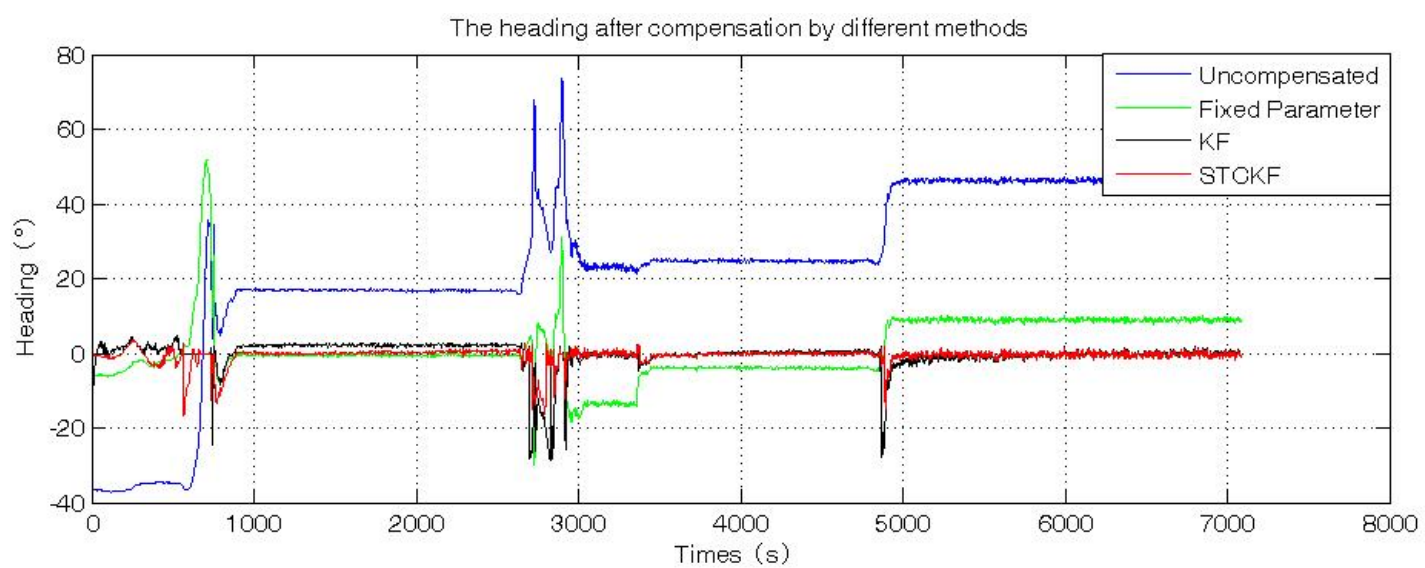

Fig.4 The heading of the compensated compass by different algorithm Tab.3 The RMSE of different compensation methods

\begin{tabular}{ccccc}
\hline & Uncompensated & Fixed Parameter & KF & STCKF \\
\hline RMSE $\left({ }^{\circ}\right)$ & 33.0189 & 8.3128 & 3.8676 & 0.8644 \\
\hline
\end{tabular}

The experimental data are treated with the Fixed Parameter, KF and STCKF, which are shown in Fig. 4. The results show that the Fixed Parameter method has certain effect of the heading compensation, but the precision is much worse. And the KF and STCKF methods have good compensation effect so that they can adapt the change magnetic field environment. However, by comparing the RMSE of the three methods in Tab. 3, we can see that the RMSE of the heading without compensation is $33.0189^{\circ}$, the RMSE of the heading compensated by Fixed Parameter and KF methods are $8.3128^{\circ}$ and $3.8676^{\circ}$, and the RMSE of the heading compensated by STCKF method is $0.8644^{\circ}$, which is much lower. It shows that the compensation method based on STCKF has better compensation precision than the methods based on Fixed Parameter and KF, when the magnetic field environment is changing.

\section{Conclusions}

In this paper a new adaptive compensation method based on strong tracking cubature Kalman filter (STCKF) is developed for heading calibration of the attitude and heading reference system (AHRS). It solves the problem that the current compensation methods cannot adapt the changing magnetic field environment and the compensation effect using Kalman filter (KF) is much worse. When the magnetic field environment changed, the method can adjust the model parameters timely so that it can ensure the heading precision of the AHRS. Thanks to the time-varying fading factor introduced in the STCKF, the weight of the current measurement is improved so that we can get much more accuracy heading. Experimental results show that adaptive compensation method based on STCKF can adapt the changing magnetic field environment very well and have the higher precision than the compensation method using Kalman filter (KF).

\section{References}

[1] K.R. Briting. Inertial Navigation System Analysis. Wiley Interscience, New York, 1971.

[2] CHEN Wei, CHEN Ruizhi, CHEN Yuwei. An adaptive calibration approach for a 2-axis digital compass in a low-cost pedestrian navigation system. Proceedings of the 2010 IEEE International Conference Instrumentation and Measurement Technology, Austin, 2010, p.1392-1397.

[3] GUO Pengfei, REN Zhang, QIU Haitao. Twelve-position calibrating method without north reference for magnetic compass. Journal of Chinese Inertial Technology. Vol. 15 (2007) No. 5, p. 598-601.

[4] LIU Shibin, YAH Jiaming, SUN Xiten. Magnetic deviation compensation for UAV'S heading measurement. Acta Aeronautica et Astronautic Sinica. Vol. 21 (2000) No. 1, p. 78-80. 
[5] B.Gambhir. Determination of magnetometer biases using module RESIDG. Computer Sciences Corporation. Report No. 3000-32700-01TN, 1975.

[6] R. Alonso, M.D. Shuster. A New Algorithm for Attitude-Independent Magnetometer Calibration. Proceedings of the Flight Mechanics/Estimation Theory Symposium. NASA-Goddard Space Flight Center, Greenbelt, MD, 1994, p. 513-527.

[7] MA Zhenhen, SHENG Wei, FANG Jiancheng. Design of micromagnetic compass integration system used on MUAV. Acta Aeronautica et Astronautica Sinica. Vol. 29 (2008) No. 4, p. 973-980.

[8] LIU Shibin. Study on automatic magnetic deviation compensation of magnetic heading measurement for UAV. Acta Aeronautica et Astronautica Sinica. Vol. 28 (2007) No. 2, p. 411-414.

[9] ZHU Jianliang, WANG Xingquan, WU Pan-long. Three-dimensional magnetic compass error compensation algorithm based on ellipsoid surface fitting. Journal of Chinese Inertial Technology. Vol. 20 (2012) No. 5, p. 562-566.

[10]Feng Yibo, Li Xisheng, Zhang Xiaojuan. Adaptive compensation method for heading error of electronic magnetic compass. Chinese Journal of Scientific Instrument. Vol. 35 (2014) No. 11, p. 2607-2611.

[11]Hine A. Magnetic Compasses and Magnetormeters. Adam Hilger LTD. London, 1968.

[12] Arasaratnam I, Haykin S, Hurd T R. Cubature Kalman filtering for continuous-discrete systems:theory and simulations, IEEE Transactions on Signal Processing, Vol. 58 (2010) No.10, p. 4977-4993.

[13]Zhou Dong-hua, XI Yu-geng, Zhang Zhong-jun. A suboptimal multiple fading extended Kalman filter. Acta Automatica Sinica. Vol. 17 (1991) No. 6, p. 689-695. 